Signal \& Image Processing : An International Journal (SIPIJ) Vol.7, No.2, April 2016

\title{
DEVELOPMENT AND HARDWARE IMPLEMENTATION OF AN EFFICIENT ALGORITHM FOR CLOUD DETECTION FROM SATELLITE IMAGES
}

\author{
Pooja Shah \\ Department of Electronics and Communication System, Nadiad, India
}

\begin{abstract}
Detecting clouds in satellite imagery is becoming more important with increasing data availability which are generated by earth observing satellites. Hence, intellectual processing of the enormous amount of data received by hundreds of earth receiving stations, with specific satellite image oriented approaches, presents itself as a pressing need. One of the most important steps in previous stages of satellite image processing is cloud detection. While there are many approaches that compact with different semantic meaning, there are rarely approaches that compact specifically with cloud and cloud cover detection. In this paper, the technique presented is the scene based adaptive cloud, cloud cover detection and find the position with assumption of sun reflection, background varying and scattering are constant. The capability of the developed system was tested using dedicated satellite images and assessed in terms of cloud percentage coverage. The system used for this process comprises of Intel $(R) X e n o n(R) C P U$ E31245 @ $3.30 \mathrm{GHz}$ processor along with MATLAB 13 software and DSPC6713 processor along with Code Compose Studio 3.1.
\end{abstract}

\section{KEYWORDS}

Satellite Images, Adaptive Cloud Detection Approach, MATLAB 13, TMS320C6713 DSK.

\section{INTRODUCTION}

Satellite images are one of the most powerful and important tools, give a good representation of what is happening at every point in the world. There is enormous image content appearing every second through multiple competing satellite systems. Manual interaction with this huge volume of data is becoming more and more inappropriate, which creates an urgent need for automatic treatment to store, organize and retrieve this content. Traditional meta-data such as geographic coverage, time of acquisition, sensor parameters, manual annotation, etc., are now insufficient to recover contents of interest when we target a specific visual concept such as desert, rock, crops, clouds or others. In many fields, we need specific contents from the satellite images as specific crops, clouds, geology structures or climate changes. Manual annotation needs to annotate every region by human where users enter descriptive word after image download from satellite. However it is a labour intensive and tedious process. Therefore we need approaches that give our intended contents automatically. 
Signal \& Image Processing : An International Journal (SIPIJ) Vol.7, No.2, April 2016

The method in this paper, segments the clouds from the background pixels and computing the position of segmented clouds which describes exact location of clouds. The system used for this process comprises of $\operatorname{Intel}(\mathrm{R}) \mathrm{Xenon}(\mathrm{R}) \mathrm{CPU} \mathrm{E} 31245$ @ $3.30 \mathrm{GHz}$ processor along with MATLAB 13 software and DSPC6713 processor along with Code Compose Studio 3.1. TMS320C6713DSK kit is the hardware backbone of this research therefore, this paper also provides key features, functional overview and board layout of DSK C6713 which is a low-cost standalone development platform. The algorithm validation is done for Spot4 satellite scenes on the Middle East from NARSS archive to determine the percent of clouds on these scenes in the period starts from January 2006 to December 2009. The different percentages of clouds coverage during each year are shown in Table 1. and Figure 1.

Table 1. Average cloud coverage though 2006 to 2009 on middle east.

\begin{tabular}{|l|l|l|l|l|}
\hline Coverage & $\mathbf{2 0 0 6}$ & $\mathbf{2 0 0 7}$ & $\mathbf{2 0 0 8}$ & $\mathbf{2 0 0 9}$ \\
\hline $0 \%(\mathrm{~A})$ & 0.44 & 0.39 & 0.43 & 0.40 \\
\hline $1 \%-10 \%(\mathrm{~B})$ & 0.08 & 0.09 & 0.07 & 0.06 \\
\hline $11 \%-25 \%(\mathrm{C})$ & 0.09 & 0.08 & 0.07 & 0.05 \\
\hline $26 \%-75 \%(\mathrm{D})$ & 0.09 & 0.08 & 0.07 & 0.06 \\
\hline $76 \%-100 \%(\mathrm{E})$ & 0.30 & 0.36 & 0.36 & 0.43 \\
\hline
\end{tabular}

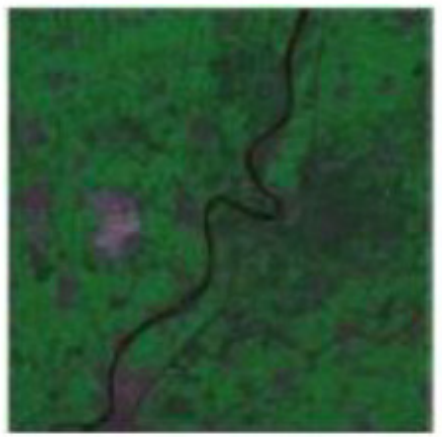

(a)

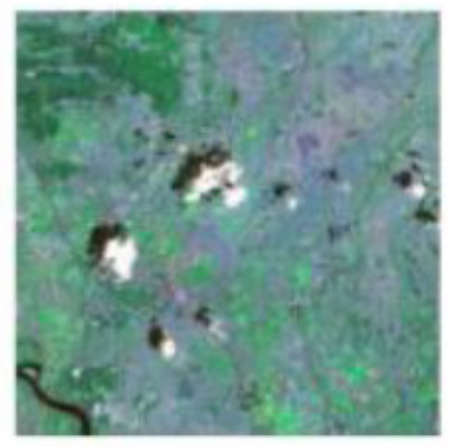

(b)

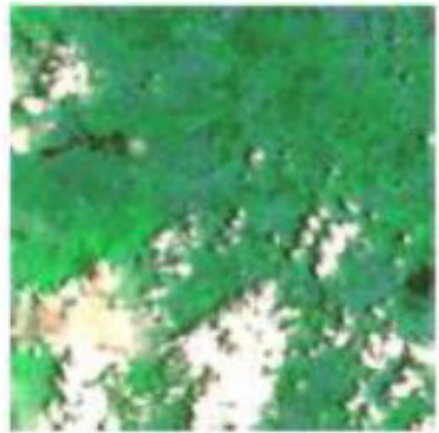

(c)

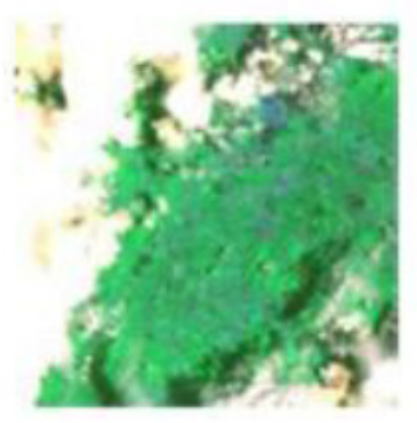

(d)

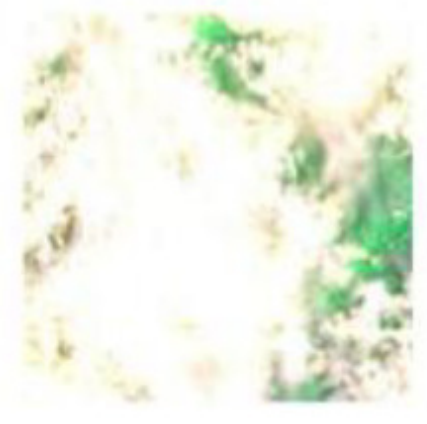

(e)

Figure 1. (a) (b) (c) (d) (e) Spot4 satellite images with different cloud coverage percentages. 
Signal \& Image Processing : An International Journal (SIPIJ) Vol.7, No.2, April 2016

\section{TMS320C6713 DSK}

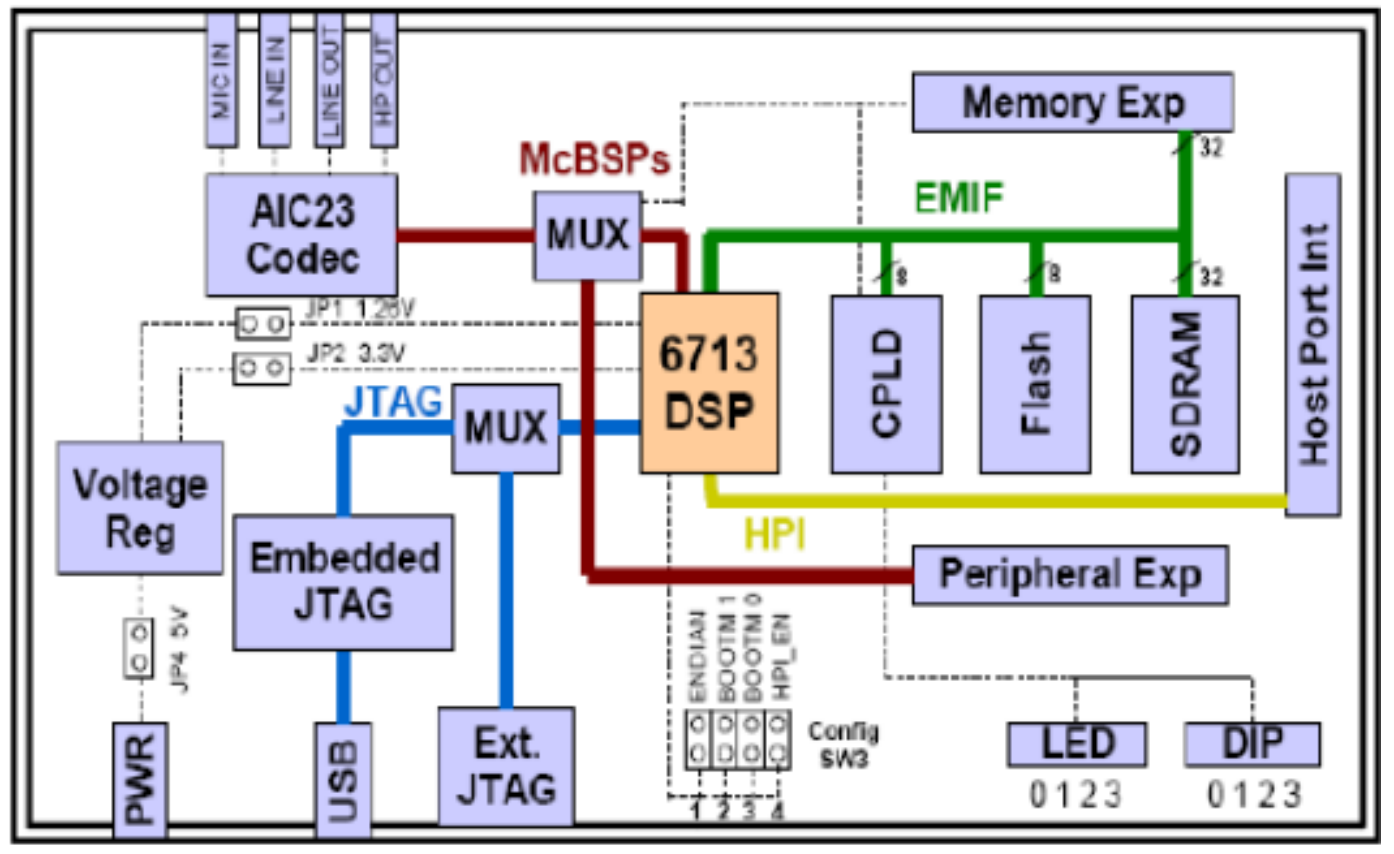

Figure 2. Block diagram of C6713DSK

The C6713 DSK is a low-cost standalone development board that enables users to evaluate and develop applications for the TI C67xx DSP family. The DSK also serves as a hardware reference design for the TMS320C6713 DSK. Schematics, logic equations and application notes are available to ease hardware development and reduce time to market. Fig.2. represents the block diagram of C6713DSK kit. The DSK comes with a full complement of on-board devices that suit a wide variety of application environments. Key features include:

- A Texas Instruments TMS320C6713 DSP operating at maximum $225 \mathrm{MHz}$

- 512 Kbytes of non-volatile Flash memory (256 Kbytes usable in default configuration)

- JTAG emulation through on-board JTAG emulator with USB host interface or external emulator

- $\quad$ Single voltage power supply $(+5 \mathrm{~V})$

External power supply of $5 \mathrm{~V}$ is used to power the board. On-board switching voltage regulators provide the $+1.26 \mathrm{~V}$ DSP core voltage and $+3.3 \mathrm{~V} \mathrm{I} / \mathrm{O}$ supplies. The board is held in reset until these supplies are within operating specifications. Code Composer communicates with the DSK through an embedded JTAG emulator with a USB host interface. The DSK can also be used with an external emulator through the external JTAG connector. The system is worked on $50 \mathrm{MHz}$ operating frequency. 
Signal \& Image Processing : An International Journal (SIPIJ) Vol.7, No.2, April 2016

\section{Cloud Detection Algorithm}

The cloud detection algorithm is adaptive thresholding based approach. In more robust algorithms, spatially and temporally varying thresholds, which better capture local atmospheric and surface effects, are used to improve their performance and broaden their application over algorithms with fixed thresholds for cloud tests. Cloud detection algorithm detects clouds , cloud cover region in satellite imagery and indentifying position with assumption of sun reflection , background varying, scattering are constant.

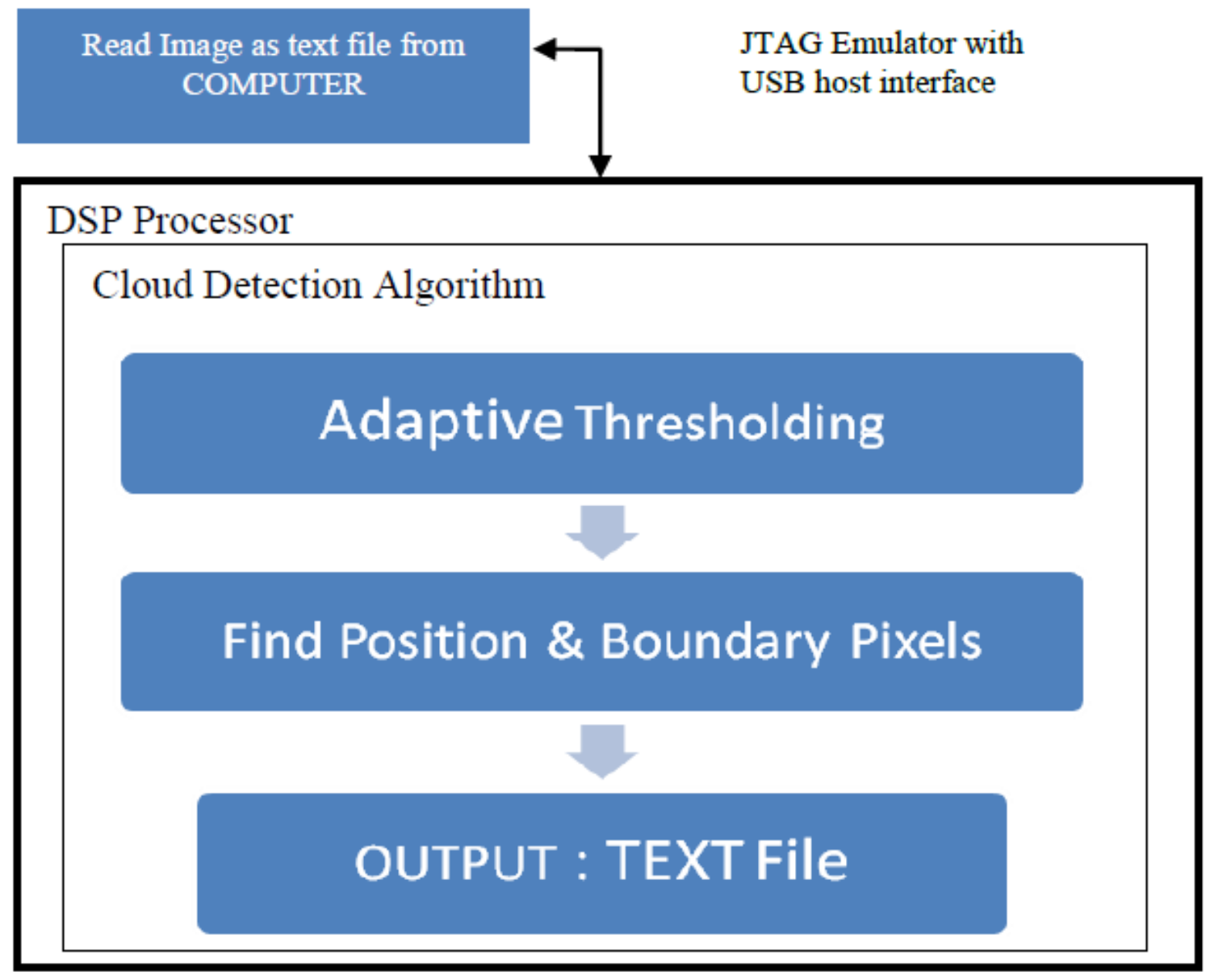

Figure 3. Block diagram of cloud detection

Implementation of cloud detection algorithm is carried out on DSP processor by reading an image as text file in CCS and results at each stage are stored as .txt file. Text files namely Thresholded image.txt, Position finding.txt and Boundary pixels.txt found at different stage of algorithm, are verified using MATLAB. 
Signal \& Image Processing : An International Journal (SIPIJ) Vol.7, No.2, April 2016

\section{DESIGN PROCESS}

The system is composed of two main stages. First stage is scene based adaptive approach which is responsible for cloud detection in each satellite images. Second stage determines where the clouds in this scene and their percentage are. This section represents steps involved in cloud detection algorithm are as follows:

\subsection{Adaptive Thresholding}

The success of most of these algorithms lies in the selection of the thresholds. The simplest method of object segmentation is called as thresholding method. Thresholding techniques are often used to segment images consisting of bright objects against dark backgrounds or vice versa.

Fixed thresholding uses a single fixed threshold for all pixels in the image and therefore works only if the intensity histogram of the input image contains distinct peaks corresponding to the desired subject and background. Hence, it cannot deal with images containing, for example, a strong illumination gradient. Also, when the background is uneven as a result of poor or nonuniform illumination conditions, a fixed level threshold will not segment the image correctly. A way to deal with such cases is to use a more sophisticated method is to different thresholds technique that is adaptive thresholding.

Adaptive thresholding, on the other hand, selects an individual threshold for each pixel based on the range of intensity values in its local neighbourhood. This allows for thresholding of an image whose global intensity histogram doesn't contain distinctive peaks. Adaptive image thresholding calculates the threshold value based on the local statistics and then applying it to the image. Thus we get more defined edges. Adaptive thresholding system outperforms fixed thresholding so; it is adapted in this work. It segments the clouds from the background pixels according to their gray value differences.

In this system adaptive thresholding is used which differentiate clouds against background. This method first calculates threshold value based on neighbouring pixels and after applying it on image it separates cloud from background. Then next step is to find the position of segmented cloud which describes exact location of cloud.

\subsection{Finding positions and boundary pixels of cloud}

Specifying the position of an object is essential in describing where actually the object is in an image. Because of the cloud some information occluded in association with low illumination and contrast areas on the ground. Thus, it's important to use efficient methods to locate cloud areas in satellite images taking in count that these areas care for special processing. In this algorithm the position of cloud is found with respect to origin of image and is used to compute cloud cover within satellite images.

Percentage of cloud cover in satellite image is given by:

$$
\text { Cloud Cove in satelite images }(\%)=\left(\frac{\text { Total no of pixels } \text { accupied bycloud }}{\text { Whole scere area }}\right) *(100)
$$


Signal \& Image Processing : An International Journal (SIPIJ) Vol.7, No.2, April 2016

The computation of cloud cover is useful to segregate cloud-free pixels and getting information from cloudy once but for that clouds needs to be detected. In our work, this cloud detection process is done by finding its boundary pixels. Object boundaries is a powerful visual cue for detecting objects in images, segmenting images into regions corresponding to individual objects. More generally, the boundaries can be used for detecting foreground object from background. Scheming boundary points on image are the exploring the use of boundaries as a bridge between foreground and background for generating candidate object locations in an input image.

\section{EXPERIMENTAL RESULTS}

The algorithm is tested on Spot4 satellite scenes with different cloud cover percents which cover about $10800 \mathrm{~km}^{2}$. Each scene covers $60 \mathrm{~km} \mathrm{x} 60 \mathrm{~km}$ of earth surface in Egypt with pixel size of $20 \mathrm{~m}$. Also on Landsat archive images database with different cloud coverage percentages. There scenes cover about $22400 \mathrm{~km}^{2}$ with $30 \mathrm{~m}$ pixel size. The different percentages of clouds coverage experimental result are shown in Table 2.

Table 2. Experimantal result in terms of average cloud coverage on middle east.

\begin{tabular}{|l|l|}
\hline Coverage & Experiment Result \\
\hline (A) & 0.04 \\
\hline (B) & 0.09 \\
\hline (C) & 0.15 \\
\hline (D) & 0.47 \\
\hline (E) & 0.91 \\
\hline
\end{tabular}

\section{RESULT ANALYSIS}

Cloud detection algorithm is simulated on Intel(R) Xenon(R) CPU E31245 @ 3.30GHz processor along with MATLAB 13 and implemented on DSPc6713 processor along with Code Composer Studio 3.1 Results tested on the image of Tropical Storm Dorian on July 24, 2013, from NOAA's GOES East satellite with 1990 x 2152 resolutions.
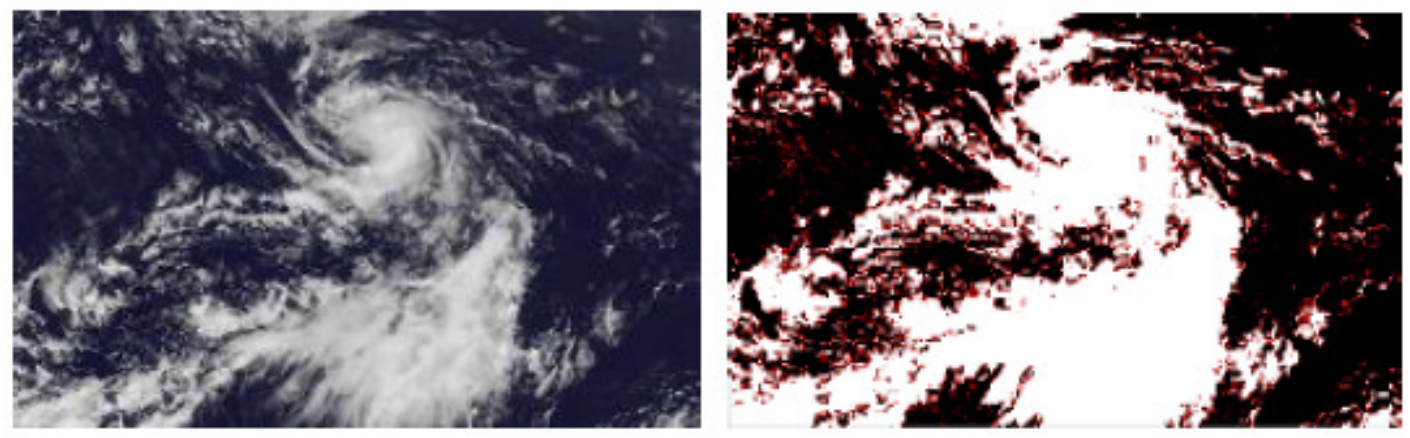

Figure 4. (a) NOAA's GOES East Satellite Image; (b) Detection of Cloud. 
Signal \& Image Processing : An International Journal (SIPIJ) Vol.7, No.2, April 2016

Cloud detection shows $89.2005 \%$ cloud covers with $72.98 \mu$ s for NOAA's GOES East satellite image in DSP processor.

Results tested on the image of Eastern U.S. Severe Weather System On Jan. 30 at 1825 UTC (1:25 p.m. EST), NOAA's GOES-13 satellite with 3600 x 3000 resolutions.
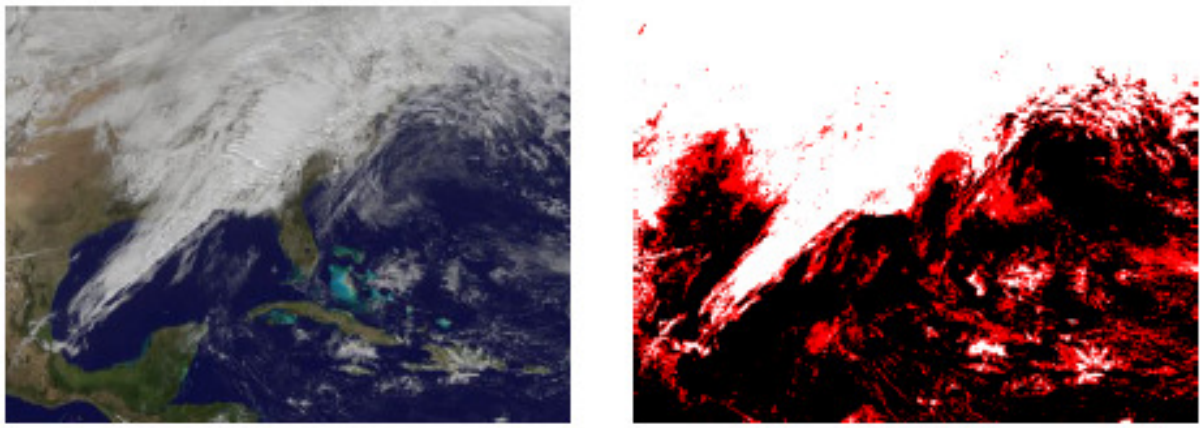

Figure 5. (a) NOAA's GOES-13 Satellite Image; (b) Detection of Cloud.

Cloud detection shows $82.6928 \%$ cloud covers with $80 \mu$ s for NOAA's GOES-13 satellite image in DSP processor.

From the above results it can be concluded that the algorithm is able to detect clouds properly and percentage of cloud cover is getting calculated accurately.

\section{CONCLUSION AND FUTURE WORK}

Cloud detection algorithm is simulated on Intel(R) Xenon(R) CPU E31245 @ 3.30GHz processor along with MATLAB 13 by using generated arithmetic function and implemented on DSPc6713 processor along with Code Composer Studio 3.1. Cloud detection algorithm obtained $89 \%$ cloud cover with $72.98 \mu$ s for NOAA's GOES satellite image of 2152 x 1990 resolutions. Algorithm achieved $82 \%$ cloud covers with $80 \mu$ s for NOAA's GOES-13 satellite image of $3600 \times 3000$ resolutions and it is able to find position of cloud. It is able to eliminate shadows (low intensity dark areas) because of reflection of cloud and detect dense clouds. The capability of the developed system was tested using dedicated satellite images and assessed in terms of cloud percentage coverage measurements. Experimental results show that the developed system enhanced the result and gives a closer assessment for cloud coverage to the real area calculations. But algorithm is unable to differentiate clouds of different bands. In future, cloud detection can be carried out with some advancement system which detects different types of clouds of different band using more robust algorithms.

\section{REFERENCES}

[1] Thiago Statella, Erivaldo Antônio da Silva, "Clouds Detection In High Resolution Images Using Mathematical Morphology" , Pecora 17 - The Future of Land Imaging...Going Operational November 18 - 20, 2008 Denver, Colorado.

[2] HARALICK, R. M., STERNBERG S. R., ZHUANG X "Image analysis using mathematical morphology”, IEEE Pattern Anal. Machine Intell. vol. PAMI-9, no. 4, pp. 532-555, Jul., 1987. 
Signal \& Image Processing : An International Journal (SIPIJ) Vol.7, No.2, April 2016

[3] Coakley, J. A., and F. P. Bretherton, (1982) "Cloud cover from high-resolution scanner data: Detecting and allowing for partially filled fields of view", J. Geophys. Res., Vol. 87, pp. 4917-4932.

[4] Lingjia Gu, Ruizhi Ren, Shuang Zhang, "Automatic Cloud Detection and Removal Algorithm for MODIS Remote Sensing Imagery”, Journal of Software, Vol. 6, No. 7, July 201.

[5] Song X N, Zhao Y S, "Cloud Detection and Analysis of MODIS Image," Geoscience and Remote Sensing Symposium, Vol.4, pp.2764-2767, 2004.

[6] Steven Platnick, Michael D King, Steven A Ackerman, et al, "The MODIS cloud products: algorithms and examples from Terra," IEEEE Transactions on Geosciences and Remote Sensing, Vol.41 (2), pp.459 - 473, 2003.

[7] Ruizhi Ren, Shuxu Guo, Lingjia Gu, "An Effective Method For the Detection and Removal of Thin Clouds from MODIS Image," SPIE Optical Engineering +Applications Satellite Data Compression, Communication, and Processing, vol.74550Z, pp.7455, 2009.

[8] Song M, Civco D L, "A Knowledge-based Approach for Reducing Cloud and Shadow," Proc. of 2002 ASPRSACSM Annual Conference and FIG22 Congress, pp.22-26, 2002.

\section{AUTHORS}

Pooja Shah receiver her B.E., in Electronics and Tele-Communication Engineering, From Gujarat Technological University, Birla Vishvakarma Mahavidhyalya, V.V.Nagar, India in 2013, and M.Tech from Dharmsinh Desai University (DDIT), Nadiad, India specializing in Nadiad, India specializing in Electronics and Communication Engineering., in 2015. She has been done her work in Space Application Centre, ISRO, Ahmedabad, India on the subject of image processing in 2015.

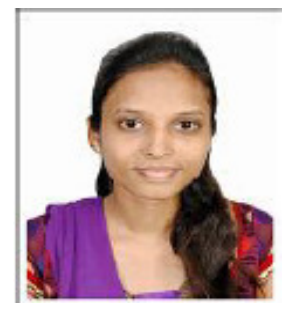

